DOI: 10.12957/demetra.2016.23266

\title{
Desenvolvimento de um Questionário de Frequência Alimentar para Populações Quilombolas do Rio Grande do Sul, Brasil
}

\section{Development of a Food Frequency Questionnaire to for Quilombola Population in Rio Grande do Sul State, Brazil}

\author{
Mariana Espinoza Rivas' \\ Pauline Müller Pacheco \\ Fernanda Souza de Bairros ${ }^{3}$ \\ Marilda Borges Neutzling² \\ 1 Universidade Federal do Rio Grande do Sul, \\ Faculdade de Medicina. Porto Alegre-RS, Brasil. \\ 2 Universidade Federal do Rio Grande do Sul, \\ Faculdade de Medicina, Programa de Pós- \\ graduação em Epidemiologia. Porto Alegre-RS, \\ Brasil. \\ ${ }^{3}$ Universidade Federal do Rio Grande do Sul, \\ Faculdade de Enfermagem, Departamento de \\ Assistência e Orientação Profissional. Porto \\ Alegre-RS, Brasil.
}

Financiamento: CNPq, Edital MCT/CNPq/MDSSAGI n 36/2010, sob o número do processo 563696/2010-9

Correspondência / Correspondence Mariana Espinoza Rivas E-mail:mari.maririvas@gmail.com

\section{Resumo}

Objetivo: desenvolver um questionário de frequência alimentar para investigar o consumo alimentar de adultos pertencentes a comunidades quilombolas do Rio Grande do Sul. Métodos: O questionário de frequência alimentar foi elaborado com base na aplicação prévia de recordatório alimentar de 24 horas de um estudo transversal realizado em 2011, com amostra representativa da população em estudo. Foram coletados 589 recordatórios, gerando uma lista de 163 itens alimentares. Destes, foram selecionados aqueles com frequência de aparecimento de pelo menos $5 \%$, além dos que apresentaram contribuição percentual para a ingestão de energia e dos nutrientes de interesse no estudo. Resultados: os alimentos mais consumidos pela população quilombola foram: arroz, óleo de soja, café, feijão, pães, açúcar e carne bovina. O alimento com maior contribuição para a ingestão calórica e de sódio foi o pão caseiro. Para a ingestão de proteína, a carne bovina. Para os lipídeos, o óleo de soja. Para as fibras e potássio, o feijão. E para a vitamina C, a laranja. A lista final do questionário de frequência alimentar contém 65 itens alimentares. Conclusões: O presente estudo contribui para a identificação dos hábitos alimentares das comunidades quilombolas do Rio Grande do Sul.

Palavras-chave: Inquéritos e Questionários. Consumo de Alimentos. Doença Crônica. Doenças Cardiovasculares. Adulto. Grupos Étnicos. 


\section{Abstract}

AIM: develop a food frequency questionnaire $(\mathrm{FFQ})$ to investigate dietary intake of adults in the maroon communities of Rio Grande do Sul. Methods: The FFQ was based on the prior application of 24-hour recalls (R24hs), a cross-sectional study conducted in 2011, with a representative sample of the maroon communities of Rio Grande do Sul (RS) surveys. 589 recalls were collected, generating a list of 163 food items. For the construction of the food list of the FFQ, we selected the items that had a frequency of occurrence of up to $5 \%$.and those that showed percentage contribution to the intake of energy and nutrients of interest in the study. Results: The most frequently consumed foods by quilombola population were rice, soybean oil, coffee, beans, breads, sugar and red meat. The food with the highest contribution to caloric intake was the homemade bread, as well as the intake of carbohydrates and sodium. The item that contributed the most to the intake of protein was red meat. For the lipids, the major contributor was soybean oil. For fibers, the largest contributor is the beans, as well as potassium. The orange was the food that contributed most to the intake of vitamin C. The final list of the food frequency questionnaire consists of 65 food items. Conclusion: This study will make an important contribution on the eating habits of the maroon communities of Rio Grande do Sul, due to the paucity of data on the subject.

Key words: Surveys and Questionnaires. Food Consumption. Chronic Disease. Cardiovascular Diseases. Adult. Ethnic Groups.

\section{Introdução}

As doenças crônicas não transmissíveis (DCNT) são consideradas um problema de saúde global e uma grande prioridade na saúde brasileira. ${ }^{1}$ Apresentam fatores de risco modificáveis em comum. Entre eles, o tabagismo, a inatividade física, o uso prejudicial de álcool e a alimentação não saudável. ${ }^{2} \mathrm{~A}$ exposição e a vulnerabilidade das populações às DCNT são também influenciadas pelas condições socioeconômicas. ${ }^{3}$ As populações vulneráveis e em desvantagem social ficam mais doentes e morrem mais cedo que as populações pertencentes a posições sociais mais elevadas. ${ }^{4}$

Entre as populações consideradas em vulnerabilidade social, estão as comunidades quilombolas, que têm recebido ultimamente maior atenção com relação aos programas sociais e de combate à fome. ${ }^{5}$ Entretanto, há uma escassez de dados sobre o padrão de consumo alimentar nessas populações. 
Para obter essas informações é necessário um instrumento específico, capaz de avaliar a ingestão dietética e distinguir diferentes padrões de consumo entre os indivíduos. O questionário de frequência alimentar (QFA) é o método de investigação do consumo alimentar mais indicado em estudos epidemiológicos que visam relacionar a dieta com a ocorrência de DCNT. ${ }^{6}$

O QFA apresenta diversas vantagens, como sua praticidade e ampla utilização em estudos epidemiológicos que relacionam a dieta com o desenvolvimento de doenças crônicas não transmissíveis. ${ }^{7}$ É uma ferramenta simples e econômica, capaz de distinguir diferentes padrões de consumo entre os indivíduos. Uma grande vantagem do QFA é a eficiência em medir o consumo habitual de alimentos, pois fornece uma informação global da ingestão em um período de tempo, em vez de medir a ingestão alimentar durante vários dias. ${ }^{6}$

Dessa forma, o objetivo do presente estudo foi desenvolver um questionário de frequência alimentar para investigar o consumo alimentar de adultos pertencentes a comunidades quilombolas do Rio Grande do Sul.

\section{Métodos}

\section{População em estudo}

O QFA foi elaborado com base na aplicação prévia de inquéritos recordatórios de 24 horas, de um estudo transversal ${ }^{8}$ realizado em 2011, com amostra representativa das comunidades quilombolas do Rio Grande do Sul (RS).

A amostra foi calculada considerando a estimativa de prevalência de 9,5\% de insegurança alimentar moderada e grave na população negra do Estado Rio Grande do Sul com erro de 3 pontos percentuais, intervalo de confiança de 95\% (IC95\%) e efeito de delineamento de 1,5. Foram acrescidos $10 \%$ ao número obtido, para perdas, o que resultou numa amostra de 634 famílias. Responderam à pesquisa todos os indivíduos, maiores de 18 anos de ambos os sexos, identificados como responsáveis pelo domicílio.

\section{Coleta de dados}

Foram coletados 589 recordatórios alimentares de 24 hs (R24hs), um para cada responsável pela família. A fim de minimizar vieses na coleta de dados, os entrevistadores foram submetidos a treinamento e com a padronização dos procedimentos de coleta de dados.

Os participantes do estudo mencionaram, através dos R24hs, todos os alimentos e bebidas consumidos ao longo do dia anterior, com suas respectivas porções, em medidas caseiras, que foram obtidas com o auxílio de um álbum fotográfico. 
Além dos R24hs, foram aplicados questionários padronizados com 120 perguntas sobre a situação socioeconômica e de segurança alimentar e nutricional das famílias. Os entrevistados também tiveram seu peso e estatura aferidos por equipe treinada e supervisionada.

O projeto foi aprovado pelo Comitê de Ética em Pesquisa da Universidade Federal do Rio Grande do Sul em 24/3/2011 - sob o número 20041/2011.Os dados foram coletados após consentimento informado dos sujeitos e anuência antecipada dos líderes das comunidades.

\section{Desenvolvimento do QFA}

Através da aplicação dos recordatórios de 24h, foi gerada uma lista com 163 itens alimentares. Para a construção da lista de alimentos do QFA, foram selecionados aqueles que tiveram uma frequência de aparecimento de pelo menos $5 \%$, ou seja, os citados em pelo menos $5 \%$ dos recordatórios de $24 \mathrm{~h}$.

A seguir, foi calculada, através da fórmula de Block et al., ${ }^{9}$ a contribuição percentual dos itens alimentares para ingestão de energia e dos seguintes nutrientes: proteína, lipídeos, carboidratos, vitamina C, fibras, sódio e potássio, que são considerados fatores de risco ou proteção para as DCNT. ${ }^{10}$ Foram selecionados os itens alimentares que contribuíram com $90 \%$ da ingestão de energia e desses nutrientes.

Foram também incluídos na lista do QFA 4 itens alimentares que não foram escolhidos através da frequência de aparecimento, mas que são de consumo da maior parte da população, inclusive em outras épocas do ano, uma vez que os R24hs foram aplicados durante o inverno. Os itens inseridos foram: melancia, melão, milho verde e abóbora.

Ao final do QFA também foram adicionadas algumas questões sobre práticas usuais no consumo de gordura, como tipo de gordura utilizada nas preparações e ingestão habitual de gordura visível ou pele das carnes. ${ }^{11}$

Os itens alimentares da lista final foram agrupados de acordo com o Guia Alimentar para a População Brasileira, publicado em 2008, ${ }^{12}$ em: cereais, tubérculos e raízes; frutas, legumes e verduras; feijões e outros alimentos ricos em proteína; leites e derivados; carnes e ovos; e gorduras e açúcares.

O presente QFA contém oito frequências de consumo, de acordo com o proposto por Roseli Sichieri: ${ }^{13}$ mais de 3 vezes por dia; 2 a 3 vezes por dia; 1 vez por dia; 5 a 6 vezes por semana; 2 a 4 vezes por semana; 1 vez por semana; 1 a 3 vezes por mês; e nunca ou quase nunca.

As porções de consumo foram estabelecidas de acordo com a média das porções referidas nos R24hs para cada alimento. Para alguns alimentos, também foi utilizada a porção convencional, como "unidade" para ovos e pão. 
Para o cálculo da composição nutricional de cada item alimentar, foi usado o software de nutrição ADS Nutri. Os alimentos e preparações não registrados no programa foram inseridos, com o auxílio da Tabela Brasileira de Composição de Alimentos - $\mathrm{TACO}^{14}$ e da tabela norte-americana da USDA, ${ }^{15}$ que foi utilizada somente para alimentos ausentes na TACO, e não suplementados e enriquecidos, uma vez que são comuns na dieta norte-americana, diferentemente da dieta brasileira.

\section{Resultados}

A população estudada foi composta por 589 adultos, sendo 362 mulheres (64,9\%) e 207 homens $(35,1 \%)$. A média de idade dos participantes era 45 anos $( \pm 17)$, a maioria com escolaridade de 4 a 8 anos de estudo completos (43,0\%) e pertencente à classe econômica C (48,2\%).

No que se refere ao estado nutricional, foi observado que a maioria da população em estudo apresenta excesso de peso $(62 \%, \mathrm{n}=353)$.

Os alimentos com frequência de aparecimento de pelo menos $5 \%$ estão listados na tabela 1. Destacam-se o arroz, o óleo de soja, o café, o feijão, os pães, o açúcar e a carne bovina, itens consumidos por mais de $40 \%$ da população.

Tabela 1. Alimentos que apresentaram frequência de aparecimento de pelo menos $5 \%$ nos R24hs. Populações Quilombolas do Rio Grande do Sul, 2011.

\begin{tabular}{l|l}
\hline Itens alimentares & $\%$ \\
\hline Arroz branco & 89,76 \\
\hline Óleo de soja & 79,57 \\
\hline Café & 69,45 \\
\hline Feijão Preto & 60,80 \\
\hline Pães & 52,94 \\
\hline Açúcar & 45,40 \\
\hline Carne bovina & 41,34 \\
\hline Leite & 37,91 \\
\hline Frango & 34,64 \\
\hline Macarrão & 31,87 \\
\hline Margarina & 29,14 \\
\hline Doces & 27,04 \\
\hline
\end{tabular}




\begin{tabular}{|c|c|}
\hline Itens alimentares & $\%$ \\
\hline Laranja & 24,95 \\
\hline Alface & 23,46 \\
\hline Refrigerante & 22,10 \\
\hline Mandioca & 20,94 \\
\hline Ovo & 19,82 \\
\hline Refresco & 18,75 \\
\hline Porco & 17,91 \\
\hline Chá & 17,11 \\
\hline Tomate & 16,33 \\
\hline Tangerina & 15,56 \\
\hline Lanches & 14,80 \\
\hline Repolho & 14,07 \\
\hline Batata cozida & 13,39 \\
\hline Queijo & 12,73 \\
\hline Mortadela & 12,09 \\
\hline Limão & 11,50 \\
\hline Biscoito maisena & 11,00 \\
\hline Sopa de legumes & 10,49 \\
\hline Biscoito cream cracker & 10,00 \\
\hline Bolo & 9,51 \\
\hline Maçã & 9,03 \\
\hline Manteiga & 8,55 \\
\hline Linguiça & 8,17 \\
\hline Bolinho frito & 7,82 \\
\hline Sopa de frango & 7,46 \\
\hline Batata frita & 7,16 \\
\hline Polenta & 6,86 \\
\hline
\end{tabular}




\begin{tabular}{l|l}
\hline Itens alimentares & $\%$ \\
\hline Salsicha & 6,56 \\
\hline Chuchu & 6,30 \\
\hline Cenoura & 6,05 \\
\hline Peixe & 5,80 \\
\hline Carreteiro & 5,58 \\
\hline Presunto & 5,35 \\
\hline Beterraba & 5,15 \\
\hline Couve & 4,96 \\
\hline
\end{tabular}

A tabela 2 exibe os principais alimentos com maior contribuição percentual para ingestão de energia e dos nutrientes de interesse.

Tabela 2. Contribuição percentual dos alimentos para a ingestão de energia e nutrientes. Populações Quilombolas do Rio Grande do Sul, 2011.

\begin{tabular}{l|l}
\hline Variáveis & Contribuição dos alimentos \\
\hline Energia (kcal) & Pão caseiro $(11,68 \%)$ \\
& Carne bovina $(10,51 \%)$ \\
& Arroz $(9,75 \%)$ \\
& Feijão $(7,24 \%)$ \\
& Óleo de soja $(5,84 \%)$ \\
\hline Proteína & Carne $(25,31 \%)$ \\
& Frango $(13,34 \%)$ \\
& Feijão $(10,04 \%)$ \\
& Carne suína $(7,90 \%)$ \\
& Pão caseiro $(7,47 \%)$ \\
\hline Lipídeos & Óleo de soja $(20,53 \%)$ \\
& Carne bovina $(20,22 \%)$ \\
& Frango $(7,98 \%)$ \\
& Margarina $(6,44 \%)$ \\
& Ovos $(3,54 \%)$ \\
\hline
\end{tabular}




\begin{tabular}{|c|c|}
\hline Variáveis & Contribuição dos alimentos \\
\hline Carboidrato & $\begin{array}{l}\text { Pão caseiro }(17,18 \%) \\
\text { Arroz }(15,91 \%) \\
\text { Açúcar }(9,87 \%) \\
\text { Feijão }(9,78 \%) \\
\text { Pão de padaria }(6,22 \%)\end{array}$ \\
\hline Fibras & $\begin{array}{l}\text { Feijão }(55,64 \%) \\
\text { Arroz }(8,64 \%) \\
\text { Pão caseiro }(6,37 \%) \\
\text { Macarrão }(3,24 \%) \\
\text { Pão de padaria }(2,90 \%)\end{array}$ \\
\hline Vitamina $\mathrm{C}$ & $\begin{array}{l}\text { Laranja }(47,71 \%) \\
\text { Bergamota }(19,32 \%) \\
\text { Limão }(7,21 \%) \\
\text { Sopa de legumes }(3,66 \%) \\
\text { Mamão }(3,37 \%)\end{array}$ \\
\hline Sódio & $\begin{array}{l}\text { Pão caseiro }(22 \%) \\
\text { Pão de padaria }(12,45 \%) \\
\text { Carne bovina }(5,77 \%) \\
\text { Polenta }(5,54 \%) \\
\text { Sopa de legumes }(4,45 \%)\end{array}$ \\
\hline Potássio & $\begin{array}{l}\text { Feijão }(21,01 \%) \\
\text { Café }(18,11 \%) \\
\text { Carne }(9,86 \%) \\
\text { Laranja }(6,79 \%) \\
\text { Frango }(4,60 \%)\end{array}$ \\
\hline
\end{tabular}

O alimento com maior contribuição percentual para energia foram os pães. Também foram os que mais colaboraram para o percentual de carboidratos e de sódio. A carne bovina foi o que mais contribuiu para o percentual de proteínas. Para os lipídeos, foi o óleo de soja. O com maior contribuição percentual para fibras e potássio foi o feijão. $\mathrm{O}$ item alimentar que mais colaborou com o percentual de vitamina $\mathrm{C}$ foi a laranja.

A lista final do QFA é composta por 65 itens alimentares, que representam 97,38\% da ingestão de calorias totais, $98,39 \%$ de lipídeos, 97,13\% de carboidratos, 97,92\% de fibras, $96,79 \%$ de vitamina C, $97,85 \%$ de sódio e $97,32 \%$ de potássio, conforme descrito na tabela 3 . 
Tabela 3. Contribuição percentual de alguns nutrientes estimados através do Questionário de Frequência Alimentar com relação ao consumo total obtido nos R24hs. Populações Quilombolas do Rio Grande do Sul, 2011.

\begin{tabular}{ll}
\hline Nutriente & Contribuição percentual \\
\hline Energia & $97,38 \%$ \\
Lipídeos & $98,39 \%$ \\
Carboidratos & $97,13 \%$ \\
Vitamina C & $96,79 \%$ \\
Sódio & $97,85 \%$ \\
Potássio & $97,32 \%$ \\
Fibras & $95,17 \%$ \\
\hline
\end{tabular}

\section{Discussão}

O desenvolvimento de um QFA específico para as populações quilombolas é importante devido à escassez de dados sobre os hábitos alimentares dessas comunidades. Por se tratar de uma população vulnerável, é importante a criação de instrumentos capazes de avaliar sua ingestão alimentar. Além disso, não foram encontrados na literatura estudos de desenvolvimento de QFA para as populações tradicionais do Brasil, o que reforça a necessidade desse tipo de estudo.

Apesar das inúmeras vantagens desse método, o presente QFA possui limitações. Uma desvantagem sua é que os R24hs foram aplicados durante o inverno, impedindo que alguns alimentos comumente consumidos em outras estações do ano fossem citados.

O presente QFA teve em sua lista final um total de 65 itens alimentares. A literatura recomenda que as listas dos QFA não contenham menos de 50 itens, pois dessa forma o consumo alimentar pode não ser corretamente avaliado, e não mais do que 100 itens, para evitar que a lista se torne muito extensa, fazendo com que o QFA perca sua característica de rapidez e simplicidade. ${ }^{6}$

Entre os alimentos mais consumidos na dieta da população quilombola estão os pães, arroz branco, café, feijão, açúcar refinado e carnes. Estes mesmos alimentos foram também os mais consumidos pelas populações de Cuiabá16 e de Niterói. ${ }^{17}$ Apesar de, atualmente, o consumo de alimentos tradicionais da dieta brasileira, como o arroz e feijão, estar diminuindo, ${ }^{18}$ estes mantiveram-se muito presentes na dieta dos quilombolas, o que pode ter impacto positivo na saúde dessa população. 
Em contrapartida, observou-se no presente estudo um baixo consumo de frutas e verduras. Esse achado talvez possa ser explicado pelo fato de as populações mais vulneráveis e com menor renda tenderem a ter um consumo menor desse grupo de alimentos. Essa tendência foi evidenciada na POF 2008-2009, ${ }^{19}$ que mostrou menor consumo de frutas e hortaliças em populações mais pobres.

Outra possível explicação para o baixo consumo de frutas e hortaliças é que em regiões mais frias, como o Estado do Rio Grande do Sul, durante o inverno, pode haver um menor consumo de alimentos desse grupo. Muitos estudos apontam que há uma variação do consumo de frutas e verduras de acordo com a época do ano, sendo maior o consumo durante o verão. Smolková et al. relatou um maior consumo de carne, carboidratos, gorduras e poucas frutas e vegetais frescos durante o inverno em relação ao verão. ${ }^{20}$

O QFA desenvolvido para a população de Porto Alegre ${ }^{21}$ é o que mais se aproxima da região pertencente à população em estudo. Apresenta uma lista de alimentos mais extensa, com concordância de grande parte dos alimentos, exceto: arroz de carreteiro, batata-doce, bolinhos fritos, farinha de mandioca, limão e carne de ovelha. Uma possível explicação é que esses alimentos fazem parte do consumo habitual das populações quilombolas, no entanto não foram identificados como sendo de consumo habitual na população urbana de Porto Alegre.

\section{ANEXO - questionário de frequência alimentar}

Nome:

Data:

Instruções de Preenchimento: Preencha o questionário a seguir, conforme sua frequência de consumo.

\begin{tabular}{|c|c|c|c|c|c|c|c|c|c|}
\hline ALIMENTO & PORÇÂO & $\begin{array}{c}\text { Mais de } \\
3 \text { vezes/ } \\
\text { dia }\end{array}$ & $\begin{array}{c}2 \text { - } 3 \\
\text { vezes/ } \\
\text { dia }\end{array}$ & $\begin{array}{c}1 \mathrm{vez} / \\
\mathrm{dia}\end{array}$ & $\begin{array}{c}5-6 \\
\text { vezes/ } \\
\text { semana }\end{array}$ & $\begin{array}{c}2 \text { - } 4 \\
\text { vezes/ } \\
\text { semana }\end{array}$ & $\begin{array}{c}1 \mathrm{vez} / \\
\text { semana }\end{array}$ & $\begin{array}{c}1-3 \\
\text { vezes/ } \\
\text { mês }\end{array}$ & $\begin{array}{c}\text { Nunca/ } \\
\text { quase } \\
\text { nunca }\end{array}$ \\
\hline Arroz & $\begin{array}{l}\text { Escumadeira } \\
\text { média cheia }\end{array}$ & & & & & & & & \\
\hline Carreteiro & $\begin{array}{l}\text { Escumadeira } \\
\text { média cheia }\end{array}$ & & & & & & & & \\
\hline Batat-doce & Unidade média & & & & & & & & \\
\hline Batata cozida & Unidade média & & & & & & & & \\
\hline Batata frita & Porção grande & & & & & & & & \\
\hline
\end{tabular}




\begin{tabular}{|c|c|c|c|c|c|c|c|c|c|}
\hline ALIMENTO & PORÇÂAO & $\begin{array}{c}\text { Mais de } \\
3 \text { vezes/ } \\
\text { dia }\end{array}$ & $\begin{array}{c}2-3 \\
\text { vezes/ } \\
\text { dia }\end{array}$ & $\begin{array}{c}1 \mathrm{vez} / \\
\text { dia }\end{array}$ & $\begin{array}{c}5-6 \\
\text { vezes/ } \\
\text { semana }\end{array}$ & $\begin{array}{c}2-4 \\
\text { vezes/ } \\
\text { semana }\end{array}$ & $\begin{array}{c}1 \mathrm{vez} / \\
\text { semana }\end{array}$ & $\begin{array}{c}1-3 \\
\text { vezes/ } \\
\text { mês }\end{array}$ & $\begin{array}{c}\text { Nunca/ } \\
\text { quase } \\
\text { nunca }\end{array}$ \\
\hline $\begin{array}{l}\text { Farinha de } \\
\text { mandioca }\end{array}$ & $\begin{array}{c}\text { Colher de sopa } \\
\text { cheia }\end{array}$ & & & & & & & & \\
\hline Macarrão & Pegador & & & & & & & & \\
\hline $\begin{array}{l}\text { Mandioca } \\
\text { cozida }\end{array}$ & Pedaço grande & & & & & & & & \\
\hline Mandioca frita & Pedaço grande & & & & & & & & \\
\hline Pão de padaria & Unidade média & & & & & & & & \\
\hline Pão caseiro & Unidade média & & & & & & & & \\
\hline Polenta & $\begin{array}{l}\text { Colher de } \\
\text { servir cheia }\end{array}$ & & & & & & & & \\
\hline Bolo & Fatia grande & & & & & & & & \\
\hline $\begin{array}{l}\text { Bolinhos fritos } \\
\text { (bolinho de } \\
\text { chuva, cueca } \\
\text { virada) }\end{array}$ & $\begin{array}{l}\text { Unidade } \\
\text { grande }\end{array}$ & & & & & & & & \\
\hline Cuca & Fatia grande & & & & & & & & \\
\hline Biscoito doce & Unidade & & & & & & & & \\
\hline $\begin{array}{l}\text { Biscoito } \\
\text { salgado }\end{array}$ & Unidade & & & & & & & & \\
\hline Abacate & $\begin{array}{l}\text { Unidade } \\
\text { pequena }\end{array}$ & & & & & & & & \\
\hline $\begin{array}{c}\text { Alface e } \\
\text { outros vegetais } \\
\text { folhosos }\end{array}$ & $\begin{array}{l}\text { Prato de } \\
\text { sobremesa } \\
\text { cheio }\end{array}$ & & & & & & & & \\
\hline Abóbora & $\begin{array}{l}\text { Escumadeira } \\
\text { média rasa }\end{array}$ & & & & & & & & \\
\hline Beterraba & $\begin{array}{l}\text { Escumadeira } \\
\text { média rasa }\end{array}$ & & & & & & & & \\
\hline Cenoura & $\begin{array}{l}\text { Colher de } \\
\text { arroz cheia } \\
\text { picada }\end{array}$ & & & & & & & & \\
\hline Couve & Folha grande & & & & & & & & \\
\hline
\end{tabular}




\begin{tabular}{|c|c|c|c|c|c|c|c|c|c|}
\hline ALIMENTO & PORÇÂA & $\begin{array}{c}\text { Mais de } \\
3 \text { vezes/ } \\
\text { dia }\end{array}$ & $\begin{array}{c}2-3 \\
\text { vezes/ } \\
\text { dia }\end{array}$ & $\begin{array}{c}1 \mathrm{vez} / \\
\text { dia }\end{array}$ & $\begin{array}{c}5-6 \\
\text { vezes/ } \\
\text { semana }\end{array}$ & $\begin{array}{c}2-4 \\
\text { vezes/ } \\
\text { semana }\end{array}$ & $\begin{array}{c}1 \mathrm{vez} / \\
\text { semana }\end{array}$ & $\begin{array}{c}1 \text { - } 3 \\
\text { vezes/ } \\
\text { mês }\end{array}$ & $\begin{array}{c}\text { Nunca/ } \\
\text { quase } \\
\text { nunca }\end{array}$ \\
\hline Chuchu & $\begin{array}{l}\text { Colher de } \\
\text { arroz cheia }\end{array}$ & & & & & & & & \\
\hline Repolho & Folha grande & & & & & & & & \\
\hline Milho verde & Unidade & & & & & & & & \\
\hline Tomate & $\begin{array}{l}\text { Unidade } \\
\text { pequena }\end{array}$ & & & & & & & & \\
\hline Abacaxi & Fatia grande & & & & & & & & \\
\hline Banana & Unidade média & & & & & & & & \\
\hline Laranja & $\begin{array}{l}\text { Unidade } \\
\text { grande }\end{array}$ & & & & & & & & \\
\hline Limão & Unidade média & & & & & & & & \\
\hline Maçã & Unidade média & & & & & & & & \\
\hline Mamão & Fatia média & & & & & & & & \\
\hline Melão & Fatia média & & & & & & & & \\
\hline Melancia & Fatia média & & & & & & & & \\
\hline Tangerina & $\begin{array}{l}\text { Unidade } \\
\text { grande }\end{array}$ & & & & & & & & \\
\hline $\begin{array}{l}\text { Sopa de } \\
\text { legumes }\end{array}$ & Prato raso & & & & & & & & \\
\hline $\begin{array}{l}\text { Feijão ou } \\
\text { lentilha }\end{array}$ & $\begin{array}{l}\text { Concha média } \\
\text { cheia }\end{array}$ & & & & & & & & \\
\hline Leite & $\begin{array}{l}\text { Copo pequeno } \\
\text { cheio }\end{array}$ & & & & & & & & \\
\hline Queijo & Fatia média & & & & & & & & \\
\hline Carne de gado & Pedaço médio & & & & & & & & \\
\hline Frango & Pedaço médio & & & & & & & & \\
\hline Ovelha & Pedaço médio & & & & & & & & \\
\hline Porco & Fatia média & & & & & & & & \\
\hline Peixe & Posta pequena & & & & & & & & \\
\hline
\end{tabular}




\begin{tabular}{|c|c|c|c|c|c|c|c|c|c|}
\hline ALIMENTO & PORÇÂAO & $\begin{array}{c}\text { Mais de } \\
3 \text { vezes/ } \\
\text { dia }\end{array}$ & $\begin{array}{c}2-3 \\
\text { vezes/ } \\
\text { dia }\end{array}$ & $\begin{array}{c}1 \mathrm{vez} / \\
\text { dia }\end{array}$ & $\begin{array}{c}5-6 \\
\text { vezes/ } \\
\text { semana }\end{array}$ & $\begin{array}{c}2-4 \\
\text { vezes/ } \\
\text { semana }\end{array}$ & $\begin{array}{c}1 \mathrm{vez} / \\
\text { semana }\end{array}$ & $\begin{array}{c}1-3 \\
\text { vezes/ } \\
\text { mês }\end{array}$ & $\begin{array}{c}\text { Nunca/ } \\
\text { quase } \\
\text { nunca }\end{array}$ \\
\hline $\begin{array}{l}\text { Embutidos } \\
\text { (linguiça, } \\
\text { mortadela, } \\
\text { salame, } \\
\text { salsicha) }\end{array}$ & Fatia média & & & & & & & & \\
\hline Ovo & Unidade & & & & & & & & \\
\hline Toucinho & Fatia média & & & & & & & & \\
\hline Sopa de frango & Prato raso & & & & & & & & \\
\hline Chimia & $\begin{array}{c}\text { Colher de sopa } \\
\text { cheia }\end{array}$ & & & & & & & & \\
\hline Doces & Unidade & & & & & & & & \\
\hline Lanches & Unidade & & & & & & & & \\
\hline $\begin{array}{l}\text { Salgadinho } \\
\text { (tipo chips) }\end{array}$ & Pacote grande & & & & & & & & \\
\hline Refrigerante & Lata & & & & & & & & \\
\hline $\begin{array}{l}\text { Maionese com } \\
\text { batata }\end{array}$ & $\begin{array}{l}\text { Colher de } \\
\text { servir cheia }\end{array}$ & & & & & & & & \\
\hline Margarina & $\begin{array}{c}\text { Colher de } \\
\text { sobremesa rasa }\end{array}$ & & & & & & & & \\
\hline $\begin{array}{l}\text { Manteiga com } \\
\text { sal }\end{array}$ & $\begin{array}{c}\text { Colher de sopa } \\
\text { rasa }\end{array}$ & & & & & & & & \\
\hline Café & $\begin{array}{l}\text { Copo pequeno } \\
\text { cheio }\end{array}$ & & & & & & & & \\
\hline Chá & Xícara & & & & & & & & \\
\hline Caldo de carne & Tablete & & & & & & & & \\
\hline Açúcar & $\begin{array}{c}\text { Colher de sopa } \\
\text { cheia }\end{array}$ & & & & & & & & \\
\hline Refresco & $\begin{array}{l}\text { Copo duplo } \\
\text { cheio }\end{array}$ & & & & & & & & \\
\hline $\begin{array}{c}\text { Sucos } \\
\text { industrializa- } \\
\text { dos }\end{array}$ & $\begin{array}{l}\text { Copo duplo } \\
\text { cheio }\end{array}$ & & & & & & & & \\
\hline
\end{tabular}


1. Que tipo de óleo/gordura você costuma usar no cozimento/preparo de refeições?

( ) Não usa

( ) Margarina

( ) Manteiga

( ) Azeite de oliva

( ) Óleo de soja/milho/outros

( ) Bacon

( ) Banha

( ) Não sabe/não cozinha

2. Quando o(a) sr.(a) come carne vermelha (boi, porco, cabrito) com gordura, o(a) sr.(a) costuma:

( ) tirar sempre o excesso de gordura

( ) comer com a gordura

( ) não come carne vermelha com muita gordura

3. Quando o(a) sr.(a) come frango/galinha com pele, o(a) sr.(a) costuma:

( ) tirar sempre a pele

( ) comer com a pele

( ) não come pedaços de frango com pele

\section{Conclusão}

No presente estudo, foi encontrada uma elevada contribuição percentual dos alimentos presentes na lista do QFA para os nutrientes em estudo. Esse fato pode ser explicado pela metodologia adotada com a colaboração percentual de energia e dos nutrientes. Outra hipótese é que haja uma monotonia alimentar por parte dessa população, com alta concordância dos alimentos mais frequentes e a contribuição calórica.

O QFA deve sempre se adaptar à população em estudo, devendo considerar seus hábitos alimentares, respeitando as variedades regionais e culturais, para retratar de forma fidedigna o consumo alimentar de cada localidade. ${ }^{6}$ Neste estudo, além da importância da construção de um questionário específico para as populações quilombolas do Rio Grande do Sul, foi possível conhecer alguns alimentos presentes no padrão alimentar desse grupo de indivíduos, sobre o qual há uma escassez de dados na literatura. O presente estudo contribui para a identificação dos hábitos alimentares das comunidades quilombolas do Rio Grande do Sul. 


\section{Agradecimentos}

A FAPERGS pela bolsa de iniciação científica e ao CNPq pelo financiamento do projeto (Edital MCT/CNPq/MDS-SAGI no 36/2010).

\section{Referências}

1. Schmidt MI, Duncan BB, Silva GA, Menezes AM, Monteiro CA, Barreto MS, et al. Chronic noncommunicable diseases in Brazil: burden and current challenges. Lancet 2011; 377(9781):1949-1961.

2. Shetty P. Nutrition transition and its health outcomes. Indian J Pediatr. 2013; 80(1):21-27.

3. World Health Organization. Comission on Social Determinants of Health. CDSH Final Report. Closing the gap in generation: health equity through action on the social determinants of health. Geneva: WHO; 2008.

4. Blas E, Kurup AS. Equity, social determinants and public health programmes. Geneva: WHO; 2010.

5. Silva DO, Guerrero AFH, Guerrero CH, Toledo LM. A rede de causalidade da insegurança alimentar e nutricional de comunidades quilombolas com a construção da rodovia BR-163, Pará, Brasil. Rev Nutr. 2008; 21:83-87.

6. Fisberg RM. Inquéritos Alimentares: métodos e bases científicos. Barueri: Manole; 2005. 334p.

7. Willet W. Future directions in the development of food-frequency questionnaires. Am J Clin Nutr. 1994; 59(1):171-174.

8. Bairros FS. Acesso aos Programas de Desenvolvimento Social e Combate à Fome, e repercussões na segurança alimentar e nutricional das comunidades quilombolas do Rio Grande do Sul [tese]. [Porto Alegre]: Faculdade de Medicina, Programa de Pós Graduação em Epidemiologia da Universidade Federal do Rio Grande do Sul; 2011.

9. Block G, Dresser CM, Hartman AM, Carroll MD. Nutrient sources in the American diet: quantitative data from the NHANES II survey. I. Vitamins and minerals. Am J Epidemiol. 1985; 122(1):13-26.

10. World Health Organization. Diet, nutrition and prevention of chronic diseases. Report FAO/WHO Expert Consulation. Geneva: WHO; 2003. 149 p. WHO Technical Report Series, n. 916.

11. Ribeiro AB, Cardoso MA. Construção de um questionário de freqüência alimentar como subsídio para programas de prevenção de doenças crônicas não transmissíveis. Rev. Nutr. 2002; 15(2):239-245.

12. Brasil. Ministério da Saúde. Coordenação Geral da Política de Alimentação e Nutrição. Departamento de Atenção Básica, Secretaria de Assistência à Saúde. Guia alimentar para a população brasileira: promovendo a alimentação saudável. Brasília: Ministério da Saúde; 2006.

13. Sichieri R. Epidemiologia da obesidade. Rio de Janeiro: Eduerj; 1998. 140 p.

14. Universidade de Campinas. Núcleo de Estudos e Pesquisas em Alimentação. Tabela brasileira de composição de alimentos. 4 ed. rev. ampl. Campinas: NEPA - UNICAMP; 2011. 161 p. 
15. U.S. Department of Agriculture. USDA National Nutrient Database for Standard Reference. Release 27. [acesso em: 8 dez. 2014). Disponível em: http://www.ars.usda.gov/Services/docs.htm?docid=25706

16. Ferreira MG, Silva NF, Schmidt FD, Silva RMVG, Sichieri R, Guimarães LV, et al. Desenvolvimento de questionário de frequência alimentar para adultos em amostra de base populacional de Cuiabá, Região Centro-Oeste do Brasil. Rev Bras Epidemiol. 2010; 13(3):413-424.

17. Anjos LA, Warlich V, Vasconcellos M, Souza D, Olinto MT, Waissmann W, et al. Development of a food frequency questionnaire in a probabilistic sample of adults from Niterói, Rio de Janeiro, Brazil. Cad Saúde Pública 2010; 26(11):2196-2204

18. Instituto Brasileiro de Geografia e Estatística. Pesquisa de Orçamentos Familiares 2008-2009: análise do consumo alimentar pessoal no Brasil. Rio de Janeiro: IBGE; 2011.

19. Instituto Brasileiro de Geografia e Estatística. Pesquisa de Orçamentos Familiares 2002-2003: análise da disponibilidade domiciliar de alimentos e do estado nutricional no Brasil. Rio de Janeiro: IBGE; 2004.

20. Smólkova B, Dusinská M, Raslová K, McNeil G, Spustová V, Blazícek P, et al. Seasonal changes in markers of oxidative damage to lipids and DNA; correlations with seasonal variation in diet. Mutat Res. 2004; 551(1-2):135-144.

21. Henn RL, Fuchs SC, Moreira LB, Fuchs FD. Development and validation of a food frequency questionnaire (FFQ-Porto Alegre) for adolescent, adult and elderly populations from Southern Brazil. Cad Saude Publica 2010; 26(11):2068-2079.

Recebido: 10/6/2016

Revisado: 19/9/2016

Aceito: $21 / 11 / 2016$ 